\title{
Research on Control Model of Flexible Manipulator for Ships Based on Fuzzy Neural Network
}

\author{
Libo Yang \\ Guangdong University of Science \& Technology , Dongguan, Guangdong, 523083, \\ China
}

Keywords: Neural network algorithm, Flexible manipulator, Fuzzy control.

\begin{abstract}
In this paper, it makes study on a kind of control model of flexible manipulator for ships based on neural network algorithm, this model can solve the operation control problem of flexible manipulator when ships are in the voyage through the combination of traditional fuzzy theory and neural network algorithm. Through simulation, it can verify that this plan can have better effect when it transports the same quality load, which is better suited to dry cargo shipping task when ships are in sailing. At the same time, it can verify the feasibility of the neural network algorithm for the control of flexible manipulator.
\end{abstract}

\section{Introduction}

Due to limited space of ships, plus considering the overall balance of ships as well as the electromagnetic interference and other factors, thus the mutual supply of ships and warships in the sea as well as material transportation becomes the difficult problem of maritime operation. It needs flexible linkage with light quality, high heavy-weight ratio, large working space and high speed, but due to the factor of physical structure of flexible manipulator, it also needs considering system elastic factor and nonlinear factor. During the process of motion, it will produce deformation such as torsion, bending, and shear, etc., which can make the modeling of nonlinear systems such as the flexible manipulator and motion control technology become relatively complicated. Due to the inaccurate measurement and modeling, plus the changes of load as well as the influence of the external disturbance, in fact, it can not get the accurate and complete motion model of the flexible manipulator, therefore, in the modeling process, it must face the existence of a large number of uncertain factors of the flexible manipulator.

This paper is mainly focused on the uncertain factors such as: the load changes of flexible manipulator, the loss of model, the uncertainty of noise and so on, then it combines the traditional control method together, namely, fuzzy control, neural network and optimal control. When the load is uncertain, the traditional method adopts PD control and optimal control and so on to control, but the control effect can not meet the high precision requirement. Aiming at solving this problem, in this paper, it adopts the combination of fuzzy neural control with PD control with optimal control as a new control method, when the load changes, it can use the fuzzy neural controller to adjust PD parameters and the optimal control parameters according to the actual load, so as to achieve better control effect, which can effectively solve the problem of load uncertainty.

\section{The Establishment of Fuzzy Neural Network Control System}

Fuzzy control is a mathematical model that does not depend on the mathematical model, which is a kind of behavior through expressing the control experience of human expert to 
determine the control. But for the complex uncertain control system, the on spot condition is often changing, human control experts can timely adjust control strategy according to the on spot condition, who also can timely sum up new experience so as to cope with the occurrence of the same situation. But as far as fuzzy control is concerned, it has not owned the self-study ability, the membership function defined by it, as well as the rules in the rule base is not adjustable, which can make the general fuzzy control have no timely adjustments under the condition of having uncertain object and occurring changes at any time, therefore, the control effect is affected. In recent years, many experts have begun to combine fuzzy control and neural network together to study them according to these features of fuzzy control. As well as the fuzzy control, the neural network does not need the accurate mathematical model of the controlled object. The neural network can adjust the network parameters constantly through self learning, by means of quantitative to express the relationship between the input signal and output signal of the controller, while the rule of fuzzy control can realize control through the qualitative summary of the experts' experience. The neural network can realize the approximation to the arbitrary function at any arbitrary precision, such feature can make it be widely used in the field of adaptive control, identification system and so on.

The general used ship's manipulator generally includes three joints, in this paper, it takes the model of three joints flexible manipulator for ships as the control object to design the system, the structure of the control system can be shown in Fig.1.

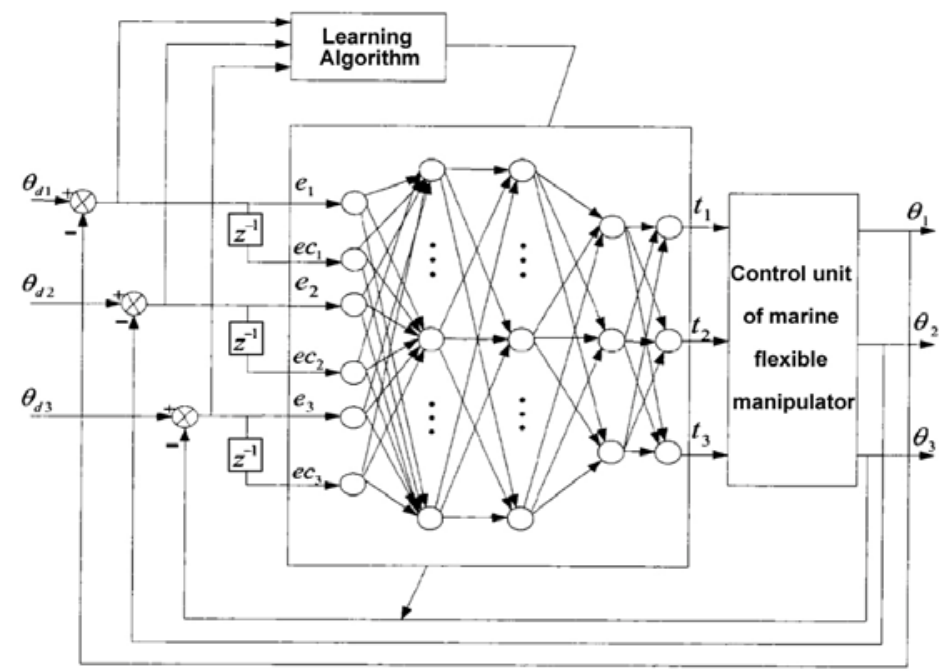

Fig.1 Control System of Flexible Manipulator for Ships Based on Fuzzy Neural Network

In Fig. $1, \theta_{d 1}, \theta_{d 2}$ and $\theta_{d 3}$ is the expected position of three joints of the manipulator, $\theta_{1}, \theta_{2}$ and $\theta_{3}$ is the actual position of the three joints, $e_{1}, e_{2}$ and $e_{3}$ is the error position of the three joints, $e c_{1}, e c_{2}$ and $e c_{3}$ is the changing rate of error, $t_{1}, t_{2}$ and $t_{3}$ is the torque of the three joints. In the figure, the fuzzy neural network is used as the joint servo controller.

In this paper, it takes the manipulator as the researching object, the structure of the designed fuzzy neural network can be shown in Fig.2. The whole controller consists of three sub-networks, each sub-network can represent the servo controller of the joint corresponding. Due to the coupling effect among the joints, the output value of the three sub-networks, namely, $u_{1}, u_{2}, u_{3}$ can times their own coupling factors, and then calculate and sum of them so as to get the final output of the network, namely, $y_{1}, y_{2}, y_{3}$.

The following is the relationship between input and output of each layer in fuzzy neural network. In the formula, ${ }^{k} \mathrm{in}_{j}^{(i)},{ }^{k} \mathrm{out} \mathrm{f}_{j}^{(i)}$ can represent the input and output of the $\mathrm{j}^{\text {th }}$ neuron of the $\mathrm{i}^{\text {th }}$ layer network in the $\mathrm{k}^{\text {th }}$ sub-net. The first layer (Level 1) introduces the input variables (error $e$ and error rate $e c$ ) into fuzzy neural network, the threshold value of input variable is $[-1,1]$. See Formula 1. 


$$
{ }^{k} \text { out }_{i}^{(1)}={ }^{k} \text { in }_{i}^{(1)}={ }^{k} x_{i}(k=1,2,3 ; i=1,2)
$$

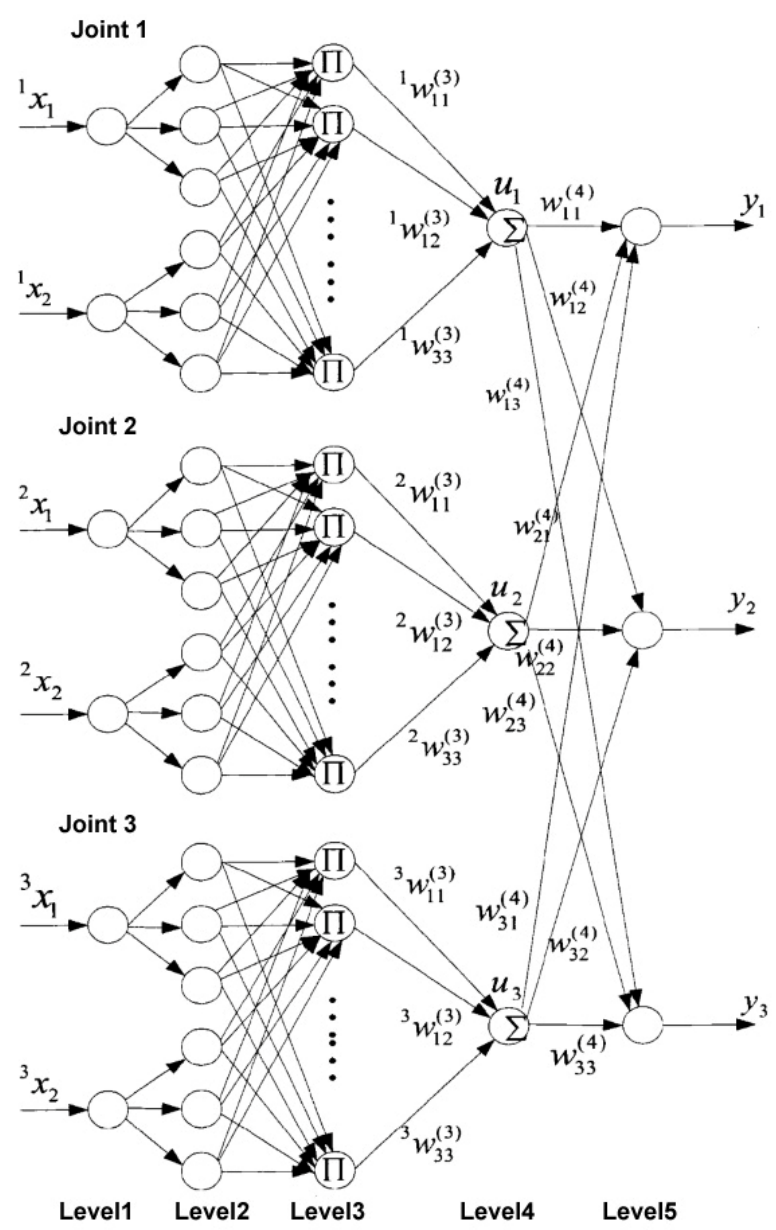

Fig.2 Manipulator Simulates Neural Network Structure

Among them, ${ }^{k} x_{i}$ represents the $\mathrm{i}^{\text {th }}$ input in the $\mathrm{k}$ sub-network.

The second layer can conduct fuzzy processing. Each input can corresponds three fuzzy language word set $\{N, Z, P\}=\{-, 0,+\}$, the corresponding membership function can adopt Gauss function. The center value of function that corresponds to $\{N, Z, P\}$ is $\{-1,0,1\}$, its width is $\{0.5,0.5,0.5\}$. The relationship between input and output of second layer can be seen in Formula 2.

$$
\begin{aligned}
& { }^{k} \text { out }_{i j}^{(2)}={ }^{k} i n_{i j}^{(2)}=\mu_{{ } A_{i}^{j}}\left({ }^{k} \text { out } t_{i}^{(1)}\right)=e^{\frac{\left({ }^{k} \text { out } t_{i}^{(1)}-{ }^{k} a_{i j}\right)^{2}}{{ }^{k} b_{i j}^{2}}} \\
& (k=1,2,3 ; i=1,2 ; j=1,2,3)
\end{aligned}
$$

Among them ${ }^{k} A_{i}^{j}$ can represent the $\mathrm{j}^{\text {th }}$ fuzzy language set in the $\mathrm{k}^{\text {th }}$ sub-network with the $\mathrm{i}^{\text {th }}$ input correspondingly, ${ }^{k} A_{i}^{j} \in\{N, Z, P\} ;{ }^{k} a_{i j} ;{ }^{k} b_{i j}$ is the center value and width of membership function of ${ }^{k} A_{i}^{j}$ respectively.

The third layer can have "and" calculation, it can use multiplication to replace the small operation, which can be seen in Formula 3

$$
{ }^{k} \text { out }_{i j}^{(3)}={ }^{k} \text { in }_{i j}^{(3)}={ }^{k} \text { out }_{1 i}^{(2)} \cdot{ }^{k} \text { out } 2 j \quad(k=1,2,3 ; i=1,2,3 ; j=1,2,3)
$$

The fourth layer adopts the method of weighted average judgment to make fuzzy operation, which can be seen in Formula 4 and Formula 5. 


$$
\begin{array}{cr}
{ }^{k} \text { in }^{(4)}=\sum_{i, j=1}^{3}\left({ }^{k} \text { out } t_{i j}^{(3)} \cdot{ }^{k} w_{i j}^{(3)}\right) & (k=1,2,3) \\
u_{k}={ }^{k} \text { out }^{(4)}={ }^{k} \text { in }^{(4)} / \sum_{i, j=1}^{3}{ }^{k}{ }^{k} t_{i j} t^{(3)} & (k=1,2,3)
\end{array}
$$

Among them, weight ${ }^{k} w_{i j}^{(3)}$ can represent the central value of the corresponding linguistic word set for the output of each control rule.

The fifth layer represents the coupling between the joints, which can be seen in Formula 6 .

$$
y_{1}=\sum_{k=1}^{3}\left(u_{k} \cdot w_{k l}^{(4)}\right) \quad(l=1,2,3)
$$

Among them, $w_{k l}^{(4)}$ can represent the coupling influence factor among the various joints.

\section{System Simulation of Manipulator}

The simulation takes the flexible arm of single rod as the example, the elastic deformation of the rod is taken as the two order mode. The parameters of the system are as follows: the length of the rod is $0.75 \mathrm{~m}$, the mass of the rod is $1.4 \mathrm{~kg}$, the stiffness of the rod is $1220 \mathrm{~N} \cdot \mathrm{m} 2$, the end load of the rod is between $0 \mathrm{~kg}$ to $5 \mathrm{~kg}$, when it is the nominal model, $M=1.4 \mathrm{~kg}$. The parameter input of joint angle of is a step signal.

The control method can respectively adopt the traditional method, namely PD control as well as the optimal control energy method, and the method proposed in this paper. Fig. 1 and Fig. 2 can respectively show the corresponding curve of $\mathrm{P}$ and rod in the first order mode and the second order mode when $M=1.4 \mathrm{~kg}$ (nominal model) and $M=5 \mathrm{~kg}$ by adopting two control methods in the two conditions respectively. It can be seen from the simulation result that when it is in the nominal model, both PD control and optimal control can make the system fast and accurate, which can effectively restrain the elastic vibration. But when the load is changed, the effect of control is not so ideal, therefore, under the non-nominal model, the amount of overshoot of the traditional method as well as steady-state time is poor. If it adopts the control method of this paper, as long as the load is changed in the load range of the training sample, both the amount of overshoot and steady-state time can achieve the desired control effect.

\section{Conclusion}

It is an important research in the field of intelligent control that ships under the complex sea conditions use flexible manipulator as the intelligent control method. Because the flexible manipulator is a very complex dynamical system, its dynamic equation has the characteristics of nonlinear, strong coupling, time variation and so on. In this paper, it adopts the neural network algorithm so as to improve the traditional fuzzy control method, which can realize the control of the flexible manipulator as well as its simulation, meanwhile the result of simulation can verify the correctness of the model. It can be regarded as a successful exploration for the application of neural network algorithm in this field.

\section{Reference}

[1] Sharma, Richa, et al. "An adaptive PID like controller using mix locally recurrent neural network for robotic manipulator with variable payload." ISA transactions 62 (2016): 258-267. 
[2] Corradini M L, Giantomassi A, Ippoliti G, et al. Robust control of robot arms via quasi sliding modes and neural networks[M]//Advances and Applications in Sliding Mode Control systems. Springer International Publishing, 2015: 79-105.

[3] Kiang, Chang Tai, Andrew Spowage, and Chan Kuan Yoong. "Review of control and sensor system of flexible manipulator." Journal of Intelligent \& Robotic Systems 77.1 (2015): $187-213$.

[4] Li-mei, CHEN Yang YAN. "The Fuzzy Optimization Control Arithmetic in Power-Plant Stream Control Boiler." Journal of Applied Science and Engineering Innovation Vol 2.1 (2015):12-15.

[5] Yong-qiu, Liu. "Design on Synchronization Control of Dual-motor in Crane."Journal of Applied Science and Engineering Innovation Vol 2.3 (2015): 71-73.

[6] Petković D, Shamshirband S, Anuar N B, et al. Input displacement neuro-fuzzy control and object recognition by compliant multi-fingered passively adaptive robotic gripper[J]. Journal of Intelligent \& Robotic Systems, 2016, 82(2): 177-187. 